\title{
Rendimiento de los puntos de corte para definir la hemorragia posparto con base en la necesidad de transfusión sanguínea
}

\author{
Omar Calvo-Aguilar ${ }^{1 *}$, Ruth Alejandra Aquino-Pérez ${ }^{2}$, Javier Vásquez-Martínez ${ }^{3}$ \\ y Sergio Arael Mendoza-Calderón ${ }^{4}$ \\ ${ }^{1}$ Subdirector de Quirófano y Área Crítica del Hospital Regional de Alta Especialidad de Oaxaca; ${ }^{2}$ Servicio de Urgencias Médico-quirúrgicas, ${ }^{3}$ Servicio de Ginecología \\ y Obstetricia, ${ }^{4}$ Servicio de Anestesiología. Hospital General Dr. Aurelio Valdivieso, Oaxaca, Oax., México
}

\section{RESUMEN}

Objetivo: Establecer el punto de corte con mejor rendimiento para la hemorragia obstétrica y la hemorragia obstétrica grave con base en la necesidad de transfusión sanguínea. Material y metodología: Estudio de tipo observacional, transversal y analítico realizado en el Hospital General «Dr. Aurelio Valdivieso» de Oaxaca, en México. La muestra se obtuvo en dos fases: en los años 2013 y 2015. La cuantificación de la pérdida sanguínea se realizó con la bolsa colectora denominada "Oaxaca». La necesidad de hemotransfusiones se estimó conforme a los lineamientos de la Guía para el uso clínico de la sangre de la Secretaría de Salud de 2008. El análisis estadístico se realizó con el software STATA 14. Los datos se presentaron como media \pm desviación estándar (SD), frecuencias y porcentajes, regresión logística múltiple para las asociaciones entre la cuantificación de pérdida sanguínea y la hemotransfusión, con intervalos de confianza (IC) del 95\%. Se realizó la curva ROC (por sus siglas en inglés, receiver operating characteristic) para comparar la capacidad de los puntos de corte en relación con la necesidad de hemotransfusión (IC 95\%). Resultados: Se incluyeron 275 mujeres. La pérdida media de sangre fue de $220.72 \mathrm{ml}$ (IC: 95\%). El punto de corte que mejor rendimiento mostró en términos de sensibilidad y especificidad fue el de $800 \mathrm{ml}$ : incluyó a todas las pacientes transfundidas y tuvo la menor cantidad de falsos positivos. Conclusiones: El punto de corte de la hemorragia posparto (HPP) en 500 $\mathrm{ml}$ tuvo una alta tasa de falsos positivos y el de $800 \mathrm{ml}$, el mejor rendimiento diagnóstico con respecto a las hemotransfusiones como estándar de oro, por lo que puede considerarse para estimar la HPP mayor, grave o severa.

Palabras clave: Hemorragia posparto. Hemorragia obstétrica. Hemorragia puerperal.

\section{Correspondence:}

*Omar Calvo-Aguilar

Aldama $\mathrm{S} / \mathrm{N}$

C.P. 71256, San Bartolo Coyotepec, Oax. México

E-mail: omarcalvoaguilar@ hotmail.com

Received for publication: 19-01-2018

Accepted for publication: 23-03-2018

DOI:10.24875/HMCM.18000103 


\section{ABSTRACT}

Objective: To establish the best cutoff point for obstetric hemorrhage and massive obstetric hemorrhage. Materials and Methods: A cross-sectional, analytical, observational study performed at the General Hospital "Dr. Aurelio Valdivieso "of Oaxaca, Mexico. The sample was obtained in two phases in 2013 and 2015. The quantification of blood loss was performed with the collection bag called "Oaxaca". The need for blood transfusions was estimated according to the Guidelines for the clinical use of blood by the Ministry of Health 2008. Statistical analysis was performed with the STATA software 14. Data were presented as mean \pm standard deviation (SD), Frequencies and percentages, multiple logistic regression for associations between quantification of blood loss and blood transfusion, with 95\% confident interval $(95 \% \mathrm{CI})$. An ROC curve was used to compare the cut-off capacity in relation to the need for blood transfusion $(95 \% \mathrm{Cl})$. Results: 275 women were included, mean blood loss was $220.72 \mathrm{ml}(\mathrm{Cl} 95 \% \mathrm{Cl})$. The cutoff point that showed the best values of sensitivity and specificity was $800 \mathrm{ml}$, includes all transfused patients and has the least false positives. Conclusions: The cutoff point of HPP in $500 \mathrm{ml}$ has high false positive rate, $800 \mathrm{ml}$ has the best diagnostic yield with respect to blood transfusions as gold standard, so it can be considered to estimate major or severe PPH. (HOSP MED CLIN MANAG. 2018;11:20-6)

Corresponding author: Omar Cavo-Aguilar, omarcalvoaguilar@hotmail.com

Key words: Pospartum hemorrhage. Obstetric hemorrhage. Puerperium hemorrhage.

\section{INTRODUCCIÓN}

Se calcula que en el mundo anualmente mueren 125,000 mujeres por una hemorragia obstétrica, por causa de una atonía uterina, alteraciones de la coagulación, alteraciones en la adhesión/inserción placentaria o lesiones traumáticas del útero y del canal obstétrico' ${ }^{1}$.

La hemorragia se clasifica conforme a las distintas definiciones propuestas por diversos organismos internacionales y sociedades médicas (Tabla 1). En el consenso la pérdida sanguínea mayor a $500 \mathrm{ml}$ debe considerarse como una HPP, según el criterio aprobado en 1989 por un grupo de trabajo de la Organización Mundial de la Salud, el mismo que ha argumentado recientemente que es una definición arbitraria y no siempre de gran importancia clínica? ${ }^{2}$.

Los criterios que se han empleado para identificar a las mujeres con una hemorragia obstétrica grave incluyen la evaluación de pruebas de laboratorio en el posparto inmediato, la alteración de constantes vitales o incluso la escala de choque hipovolémico ${ }^{3}$. En la revisión del grupo Cochrane del año 2014 se utilizó como punto de corte la pérdida sanguínea de $1,000 \mathrm{ml}$ para identificar los casos de gravedad, manifestando que existen puntos de corte alternativos como los 600 o los $1,500 \mathrm{ml}$. Tal variedad de criterios hace evidente que no existe en la actualidad un punto de corte universalmente aceptado para las pérdidas graves ${ }^{4}$.
Tabla 1. Resumen de las definiciones internacionales de hemorragia obstétrica actualmente en uso

Guía del Congreso Americano de Ginecólogos y Obstetras

- «Ninguna definición única y satisfactoria»

- Definición convencional:

- Pérdida de sangre mayor de $500 \mathrm{ml}$ después del parto vaginal

- Pérdida de sangre mayor de 1,000 ml después de la cesárea

\section{Guía Australiana}

- Pérdida de sangre mayor de $500 \mathrm{ml}$ después del parto vaginal

- Pérdida de sangre mayor de $750 \mathrm{ml}$ después de la cesárea

Sociedad Austríaca de Ginecología y Obstetricia

- Pérdida de sangre de 500 a 1,000 ml con signos clínicos de shock hipovolémico

- Pérdida de sangre mayor de 1,000 ml

Sociedad Alemana de Ginecología y Obstetricia

- Pérdida de sangre mayor de $500 \mathrm{ml}$ después del parto vaginal

- Pérdida de sangre mayor de $750 \mathrm{ml}$ después de la cesárea

Colegio Inglés de Ginecología y Obstetricia

- Pérdida de sangre de 500 a 1,000 ml

- Hemorragia obstétrica severa:

- Pérdida de sangre mayor de 1,000 ml

- Pérdida de sangre de 500 a 1,000 ml con signos clínicos de shock hipovolémico

Organización Mundial de la Salud

- Pérdida de sangre mayor de $500 \mathrm{ml}$

- Hemorragia obstétrica severa:

- Pérdida de sangre mayor de $1,000 \mathrm{ml}$

Adaptado de Rath ${ }^{11}$ 


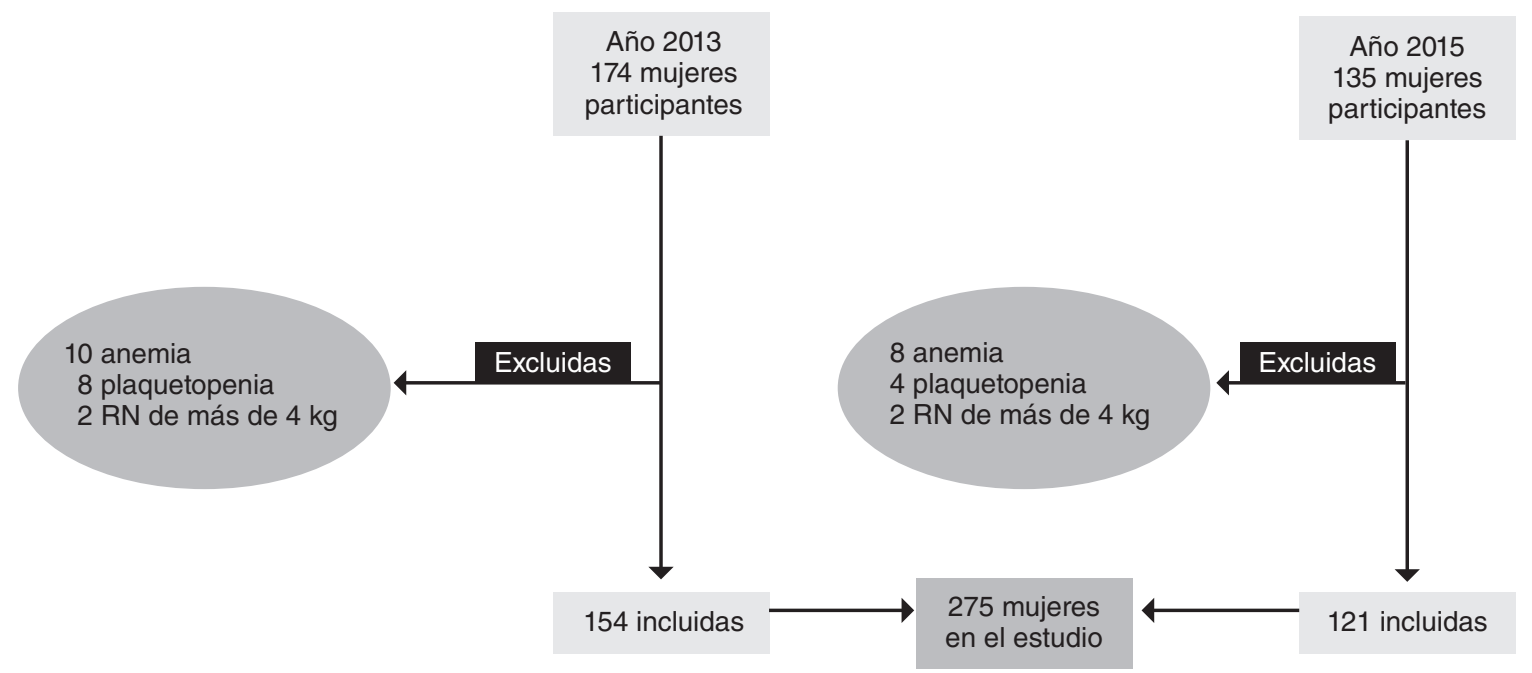

Figura 1. Esquema del proceso de captación de pacientes en las dos fases del estudio (2013 y 2015).

Los esfuerzos para determinar la incidencia de la HPP se ven obstaculizados por dos condiciones: la falta de un punto de corte establecido con fundamentos sólidos debido a la ausencia de estudios que abonen en este sentido y la dificultad para tener un instrumento de medición apropiado ${ }^{5,6}$, ya que se recomienda emplear bolsas colectoras sólo en caso de sospecha de hemorragia por factores de riesgo ${ }^{7}$.

\section{Objetivo}

Establecer el punto de corte con mejor rendimiento para la hemorragia obstétrica y la hemorragia obstétrica grave en el posparto.

\section{MATERIAL Y MÉTODO}

Estudio de tipo observacional, transversal y analítico realizado en la División de Ginecología y Obstetricia del Hospital General «Dr. Aurelio Valdivieso» de los Servicios de Salud del estado de Oaxaca, en México.

El cálculo de la muestra se obtuvo con un nivel de confianza estadística (z) del 95\%, una precisión del 3\% (d), estimando una prevalencia del $6 \%$, donde $Q$ tomó el valor de 94. Se empleó la fórmula $n=z^{2}(P Q) / d^{2}$ para estudios transversales y se obtuvieron 241 pacientes; con una estimación de pérdidas del $10 \%$, el total fue de 268 pacientes.

La muestra se obtuvo de forma aleatoria simple con números random, de enero a octubre de 2013 y de enero a noviembre de 2015; hubo un total de 309 pacientes que aceptaron participar en el estudio conforme a los principios de Helsinki, que firmaron y aceptaron luego de la explicación el consentimiento informado (Fig.1). El protocolo fue aprobado por el Comité de Ética en Investigación de la unidad hospitalaria con el número CEI of. 007/2015. Se consideró el siguiente criterio para el ingreso: mujeres con embarazos de término sin alteraciones de la coagulación ni anemia en el embarazo. Todas las pacientes firmaron el consentimiento bajo información de acuerdo con el título segundo de la Ley General de Salud vigente en México. Fueron excluidas 30 pacientes por cursar con alteraciones hematológicas (18 con anemia y 12 con trombocitopenia) y se eliminaron los datos de cuatro porque el recién nacido (RN) pesó más de 4 kg.

La cuantificación de la pérdida sanguínea en el posparto se realizó en la bolsa colectora denominada "Oaxaca», de $90 \times 60 \mathrm{~cm}$, en forma de rombo, con un receptáculo en la mitad inferior de manera que la superior sirve como medio de sujeción y la parte inferior se encuentra graduada. La colocación se llevó a cabo de forma posterior al nacimiento y corte del cordón umbilical del RN, para evitar otros líquidos. En todos los casos la atención del alumbramiento se realizó con un manejo activo utilizando oxitocina $10 \mathrm{UI}$ a la salida del hombro del RN. El uso de hemoderivados se basó en los lineamientos de la Guía para el uso clínico de la sangre de la Secretaría de Salud de 2007 y la Norma Oficial Mexicana NOM-253-SSA1-2012 para la disposición de sangre humana y sus componentes con fines terapéuticos ${ }^{8,9}$. 
Tabla 2. Características de las mujeres incluidas en el estudio

\begin{tabular}{|c|c|c|c|c|c|}
\hline Variable $(n=275)$ & Media & SD & Máx. & Mín. & IC (95\%) \\
\hline Edad & 23.72 & 5.56 & 42 & 14 & $23.07-24.38$ \\
\hline Talla & 1.54 & 0.07 & 1.72 & 1.42 & $1.53-1.54$ \\
\hline Peso & 65.63 & 9.6 & 96 & 47 & $64.49-66.76$ \\
\hline IMC & 27.56 & 3.67 & 40.42 & 18.68 & $27.12-27.69$ \\
\hline Gestaciones & 2.1 & 1.22 & 7 & 1 & $1.95-2.24$ \\
\hline Peso RN & $3,139.57$ & 353.81 & 3,950 & 2,000 & $3,097.75-3,181.38$ \\
\hline Hemorragia & 220.72 & 227.69 & 2,600 & 5 & $193.80-247.63$ \\
\hline
\end{tabular}

IMC: índice de masa corporal.

Tabla 3. Rendimiento diagnóstico conforme a los diferentes puntos de corte establecidos en el estudio para la hemorragia obstétrica masiva

\begin{tabular}{|c|c|c|c|c|}
\hline Valor en ml (punto de corte) & Sensibilidad & Especificidad & LR+ & LR- \\
\hline 500 & 100 & 93.38 & 15.11 & 0 \\
\hline 525 & 100 & 95.22 & 20.92 & 0 \\
\hline 550 & 100 & 95.59 & 22.66 & 0 \\
\hline 600 & 100 & 97.43 & 38.85 & 0 \\
\hline 650 & 100 & 98.16 & 54.4 & 0 \\
\hline 750 & 100 & 98.53 & 68 & 0 \\
\hline 800 & 100 & 99.26 & 135.99 & 0 \\
\hline 900 & 66.67 & 99.26 & 90.66 & 0.33 \\
\hline 1,150 & 66.67 & 100 & 0 & 0.33 \\
\hline 2,600 & 33.33 & 100 & 0 & 0.66 \\
\hline
\end{tabular}

LR: razón de verosimilitud.

\section{Análisis estadístico}

El análisis estadístico se realizó con el software STATA 14. Los datos se presentaron como media \pm SD para las variables continuas y como frecuencias y porcentajes para las categóricas. Se empleó el análisis de regresión logística múltiple para evaluar las asociaciones entre la cuantificación de la pérdida sanguínea y la necesidad de hemotransfusión, obteniendo también el cálculo los IC del 95\%. Se realizó la curva ROC de las características operativas del receptor con la descripción del área bajo la curva con IC del 95\%, para comparar la capacidad de los puntos de corte propuestos en relación con la necesidad de transfusión sanguínea.

\section{RESULTADOS}

Se recabaron los datos de 275 mujeres que concluyeron el parto con un RN vivo; 154 de ellas fueron ingresadas en el año 2013 durante la primera fase y 121, durante la segunda en el año 2015.

En la tabla 2 se puede observar la medición, que incluyó aquellas características que pueden influir en la pérdida sanguínea durante el parto, como son el peso materno (sobrepeso/obesidad), el número de hijos y el peso del $\mathrm{RN}$. La muestra estuvo integrada por mujeres jóvenes con una media de 23.72 años, pero también por mujeres en los extremos de la edad reproductiva. El número promedio de gestaciones fue de 2.1; se observó que 112 mujeres cursaron su primer embarazo, 106, el segundo embarazo y 57 tuvieron más de tres hijos (multigestas). En 29 mujeres hubo antecedente de cesárea en algún embarazo previo y en 16, antecedente de aborto; sólo una mujer refirió haber presentado un nacimiento prematuro como antecedente de importancia.

Por otra parte, con base en las mediciones obtenidas de la cuantificación sanguínea, se realizó la evaluación 


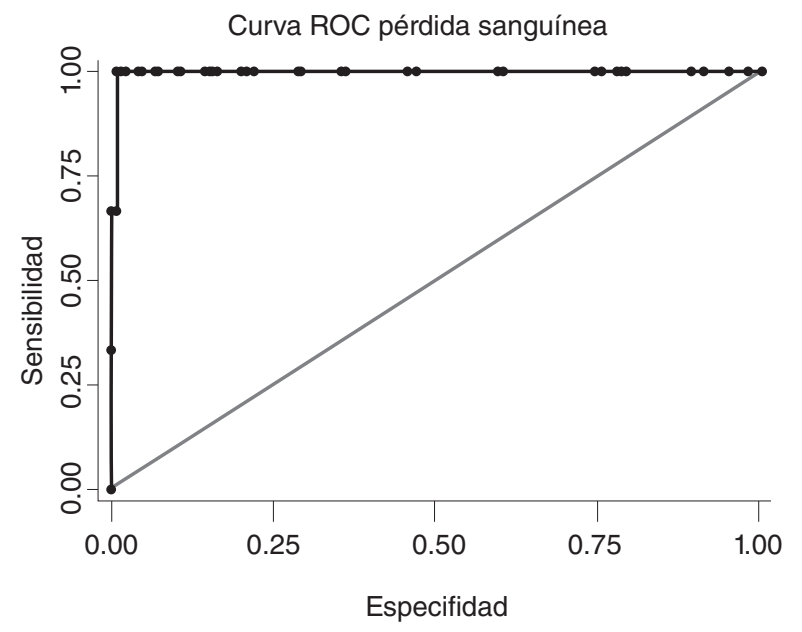

Área bajo la curva 0.9975

Figura 2. Curva ROC de pérdida sanguínea basada en la necesidad de transfusión para el diagnóstico de hemorragia posparto.

de los diferentes puntos de corte para obtener valores de sensibilidad y especificidad, razones de verosimilitud positivas y negativas en relación con la necesidad de transfusiones sanguíneas (estándar de oro) a partir del valor considerado actualmente como punto de corte oficial: $500 \mathrm{ml}$ (Tabla 3).

La pérdida media de sangre obtenida fue de $220.72 \mathrm{ml}$ (IC: 95\%). Tres mujeres de la muestra requirieron una transfusión sanguínea de acuerdo con los criterios del lineamiento técnico para transfusiones de México. El análisis mostró que el área bajo la curva fue del 99.7\% y el punto de corte que mostró mejores valores de sensibilidad y especificidad fue el de $800 \mathrm{ml}$, ya que incluyó a todas las transfundidas y tuvo la menor cantidad de falsos positivos al comparar con el resto de puntos de corte propuestos. Los datos se muestran en la figura 2.

Se puede observar, conforme a los valores obtenidos, que el percentil 99 tuvo un valor de $960 \mathrm{ml}$, con el inconveniente de excluir a una paciente transfundida, en tanto que el valor obtenido para el percentil 95 fue de $550 \mathrm{ml}$, con la ventaja de incluir a todas las mujeres transfundidas pero el inconveniente de su poca especificidad, pues incluyó muchos falsos positivos. El percentil 50 obtuvo un valor de $150 \mathrm{ml}$, que se encuentra por debajo de la media de $220 \mathrm{ml}$, de manera que es poco útil para la toma de decisiones. El empleo de 2
SD de la media obtuvo un valor de $468.35 \mathrm{ml}$, cercano al estimado de $500 \mathrm{ml}$.

La curva de las características operativas del receptor muestra que el área bajo la curva fue de 0.997, conforme a las tres mujeres que requirieron de transfusiones sanguíneas; los IC obtenidos fueron de 0.979 a 0.999 . Los valores de sensibilidad y especificidad de los diferentes puntos de corte obtenidos de la cuantificación de la pérdida sanguínea que se muestran en la tabla 2 permiten observar que el valor de $800 \mathrm{ml}$ se ubica como el mejor punto de corte para definir la hemorragia posparto masiva por la necesidad de transfusión sanguínea debido a que es el punto donde se obtienen los valores más altos de sensibilidad y especificidad. Respecto a los puntos de corte de 500 y $1,000 \mathrm{ml}$, el primero se encuentra con valores muy bajos y el segundo no incluye a todas las mujeres con necesidad de ser transfundidas.

\section{DISCUSIÓN}

La HPP actualmente es la principal causa de muerte materna y morbilidad materna extrema, así como la enfermedad más costosa por encontrarse asociada al uso de hemoderivados, cirugías y hospitalización en unidades de cuidados intensivos ${ }^{10}$. Recientemente se ha despertado el interés por establecer el momento en el que se «inicia» la HPP. Las definiciones basadas en la estimación de la pérdida de volumen tienen el inconveniente de la medición, dado que las estimaciones visuales carecen de exactitud, por subestimar las pérdidas en el $46-75 \%$ de los casos, y se observa también en los análisis que la diferencia entre la estimación visual y la medición es significativa $8,11,12$.

El uso de bolsas colectoras ha mostrado ser una herramienta eficaz en la cuantificación de las pérdidas sanguíneas si se compara con la espectrofotometría como el estándar de oro ${ }^{9}$, por lo que se puede considerar su uso de manera constante, y no sólo en los casos de riesgo, puesto que la HPP no es un evento previsible. Por ese motivo es importante determinar el límite normal de pérdida sanguínea en el posparto para iniciar las maniobras de reanimación (fin terapéutico), establecer la auditoría (prevalencia de la enfermedad) o con fines académicos ${ }^{8}$. La medición mediante una bolsa graduada permite medir de manera 
más objetiva la pérdida sanguínea durante el posparto inmediato. Este dispositivo, de fácil uso, se coloca después del alumbramiento, para evitar la mezcla con líquido amniótico, orina o soluciones. Además, al colocar líneas de decisión en los valores se podrían acompañar las medidas establecidas para el manejo de la hemorragia obstétrica con el fin de que las maniobras no se demoren y la atención sea oportuna.

El rendimiento diagnóstico del punto de corte en 800 $\mathrm{ml}$ ofreció mejores resultados que los diferentes puntos de corte propuestos en el escenario internacional, como 500, 1,000 o 1,200 ml. En la revisión de HERA se definió la HPP menor como la pérdida de más de $500 \mathrm{ml}$ de sangre y la HPP mayor como la pérdida de más de 1,000 ml, estimando una prevalencia de HPP del $6.09 \%{ }^{13}$. En la presente investigación, con el mismo punto de corte, la prevalencia fue del $6.1 \%$. Sin embargo, los autores de la revisión Cochrane indican que cuando la pérdida de sangre se mide objetivamente, la prevalencia puede incrementar hasta el $10.6 \%{ }^{4}$. Estos mismos autores también observaron que la prevalencia de HPP severa (definida como una pérdida de sangre $>1,000 \mathrm{ml}$ ) era del $1.86 \%$, cifra que contrasta con los datos obtenidos en la presente revisión, en la que se obtuvo un $0.72 \%$ con el mismo punto de corte. Basándose en la necesidad de transfusiones sanguíneas, la prevalencia de HPP severa es del $1.09 \%$, en tanto que si se establece con base en la cantidad de $800 \mathrm{ml}$ (el mejor rendimiento) la prevalencia se encuentra en el 2.18\%, situación descrita en la revisión de Kerr, et al. referente a la duplicación de la prevalencia cuando la pérdida de sangre se mide objetivamente (3.04\%; IC 95\%: 2.90-3.17) ${ }^{4}$. Por su parte, el percentil 99 ubicado en $960 \mathrm{ml}$ tuvo el inconveniente de excluir a una de las mujeres que requirió una transfusión sanguínea.

La utilización como estándar de oro de las transfusiones sanguíneas se fundamenta en la utilidad clínica que tiene el empleo de hemoderivados en el caso de la HPP, considerando éste como el evento más relevante en la resolución de la complicación, además de ser una intervención que acompaña a la histerectomía obstétrica ${ }^{8,14}$. El IC 95\% obtenido en la presente revisión alcanza un valor cercano a los $500 \mathrm{ml}$, cantidad que bien podría considerarse un valor de referencia para la preparación de intervenciones preventivas en la HPP (uso de uterotónicos, solicitud de unidades de sangre disponibles, disponibilidad de carro rojo, etc.), y el punto de corte de $800 \mathrm{ml}$ (HPP mayor o severa) podría considerarse el punto de inicio de maniobras de reanimación e intervención del código rojo con el objetivo de prevenir hemorragia masiva obstétrica $(\mathrm{HMO})$, que se define como la pérdida mayor a 2,500 $\mathrm{ml}$ de sangre y se asocia a una morbilidad significativa, a la necesidad de ingreso en unidades de pacientes críticos y a la realización de una histerectomía obstétrica ${ }^{15}$.

La necesidad de establecer un punto de corte obedece, como bien establecieron Kerr, et al. en el año 2016, a un objetivo terapéutico, dada la utilidad clínica del inicio de la reanimación en la HPP mayor, puesto que el demorar el inicio de la terapéutica o el actuar a destiempo tienen repercusiones graves; sin embargo, no se debe olvidar la vía de nacimiento, ya que en los casos de cesárea la pérdida es mayor que en los de parto eutócico 8,16,17

El estudio cuenta con limitaciones que deben ser consideradas; la primera y más importante radica en que la cuantificación sólo es posible de manera eficaz en mujeres de posparto, y no en el caso de las cesáreas. Además, la muestra se limitó a 275 mujeres todas ellas sanas, o consideradas como de bajo riesgo, dado que el estudio no contó con financiamiento específico, motivo por el que fue realizado en dos fases, además de por la problemática de la atención propia de un hospital de segundo nivel con sobreocupación (115\%).

\section{CONCLUSIÓN}

El punto de corte de la HPP en $500 \mathrm{ml}$ tiene el inconveniente de presentar una alta tasa de falsos positivos, lo que impide la optimización de recursos humanos y materiales destinados a la reanimación.

El punto de corte en $800 \mathrm{ml}$ empleando como estándar de oro la necesidad de transfusiones sanguíneas obtuvo los mejores valores de sensibilidad y especificidad, motivo por el que puede ser considerado el de mejor rendimiento para estimar la HPP mayor, grave o severa.

\section{BIBLIOGRAFÍA}

1. Mclintock $\mathrm{C}$. James $\mathrm{AH}$. Obstetric hemorrhage. J Thromb Haemost. 2011;9(8):1441-51

2. Kerr R, Eckert LO, Winikoff B, et al. Pòkòkostpartum haemorrhage: Case definition and guidelines for data collection, analysis, and presentation of immunization safety data. Vaccine. 2016;34(49):6102-9. 
3. Nyfløt LT, Sandven I, Stray-Pedersen B, Pettersen S, Al-Zirqi I, Rosenberg $\mathrm{M}$, et al. Risk factors for severe postpartum hemorrhage: a case-control study. BMC Pregnancy and Childbirth. 2017;17(1):17.

4. Mousa HA, Blum J, Abou El Senoun G. Shakur H, Alfirevic Z. Treatment for primary postpartum haemorrhage. Cochrane Database Syst Rev. 2014;(2):CD003249.

5. World Health Organization, Recomendations for the Prevention and Treatment of Postpartum Haemorrhage. 2012. [Internet] Disponible en: http://apps.who.int/iris/bitstream/10665/75411/1/9789241548502_ eng.pdf.

6. Abdul-Kadir R, McLintock C, Ducloy AS, El-Refaey H, England A, Federici $A B$, et al. Evaluation and management of postpartum hemorrhage: consensus from an international expert panel. Transfusion. 2015;55(3):691.

7. Sentilhes L, Vayssière C, Deneux-Tharaux C, Aya AG, Bayoumeu F, Bonnet $M P$, et al. Postpartum hemorrhage: guidelines for clinical practice from the French College of Gynaecologists and Obstetricians (CNGOF): in collaboration with the French Society of Anesthesiology and Intensive Care (SFAR). Eur J Obstet Gynecol Reprod Biol. 2016;198:12-21.

8. Secretaría de Salud. Guía para el uso clínico de la sangre. 3. ${ }^{a}$ ed. 2007. [Internet] México. Editorial: Secretaria de Salud, Asociación Mexicana de Medicina Transfusional y Agrupación Mexicana para el estudio de la Hematología A.C. Disponible en: http://www.ammtac.org/docs/GuiasTransfusion/ GuiaParaEIUsoClinicoDeLaSangre.pdf.
9. Norma Oficial Mexicana NOM-253-ssa1-2012, para la disposición de sangre humana y sus componentes con fines terapéuticos (modifica la NOM-003ssa2-1993, publicada el 18 de julio de 1994).

10. Kerr RS, Weeks AD. Postpartum haemorrhage: a single definition is no longer enough. BJOG. 2017;124(5):723-6.

11. Patel A, Goudar SS, Geller SE, Kodkany BS, Edlavitch SA, Wagh K, et al. Drape estimation Vs. Visual assessment for estimating postpartum hemorrhage. Int J Gynaecol Obstet. 2006;93(3):220-4.

12. Rath WH. Postpartum hemorrhage- update on problems of definitions and diagnosis. Acta Obstet Gynecol Scand. 2011;90(5):421-8.

13. Vendittelli F Barasinski C, Pereira B, Dreyfus M, Lémery D, Bouvier-Colle MH, et al. Policies for management of postpartum haemorrhage: The HERA crosssectional study in France. Eur J Obstet Gynecol Reprod Biol. 2016;205:21-6.

14. Conner SN, Tuuli MG, Colvin R, Shanks AL, Macones GA, Cahill AG. Accuracy of Estimated Blood Loss in Predicting Need for Transfusion after Delivery. Am J Perinatol. 2015;32(13):1225-30.

15. Guasch E, Gilsanz F. Massive obstetric hemorrhage: Current approach to management. Med Intensiva. 2016:40(5):298-310.

16. Feldman R, Wang EY, Bastek JA, Sirinivas SK. Assessing reVITALize: shlould the definition of postpartum hemorrhage differ by mode of delivery. Am J Perinatol. 2017:34(5):503-7.

17. Mclintock C, James H. Obstetric hemorrhage. J Thromb Haemost. 2011;9(8):1441-51. 